\title{
A dusty pinwheel nebula around the massive star WR 104
}

\author{
Peter G. Tuthill, John D. Monnier \& William C. Danchi \\ Space Sciences Laboratory, University of California, \\ Berkeley, CA, 94720-7450
}

\begin{abstract}
Wolf-Rayet (WR) stars are luminous massive blue stars thought to be immediate precursors to the supernova terminating their brief lives. The existence of dust shells around such stars has been enigmatic since their discovery some 30 years ago; the intense radiation field from the star should be inimical to dust survival [1]. Although dust-creation models, including those involving interacting stellar winds from a companion star [2], have been put forward, high-resolution observations are required to understand this phenomena. Here we present resolved images of the dust outflow around Wolf-Rayet WR 104, obtained with novel imaging techniques, revealing detail on scales corresponding to about $40 \mathrm{AU}$ at the star. Our maps show that the dust forms a spatially confined stream following precisely a linear (or Archimedian) spiral trajectory. Images taken at two separate epochs show a clear rotation with a period of $220 \pm 30$ days. Taken together, these findings prove that a binary star is responsible for the creation of the circumstellar dust, while the spiral plume makes WR 104 the prototype of a new class of circumstellar nebulae unique to interacting wind systems.
\end{abstract}

Observations of WR 104 were made with the Keck I telescope on 14 April and 4 June 1998, and employed the technique of aperture masking interferometry in order to recover information out to the diffraction limit of the $10 \mathrm{~m}$ Keck aperture [3], 幽. We present, in Figure 1, reconstructed images taken at $1.65 \& 2.27 \mu \mathrm{m}(\Delta \lambda=0.33 \& 0.16 \mu \mathrm{m}$ respectively $)$ for both observing epochs. As infrared emission from the hot circumstellar dust dominates the infrared region of the spectrum, we may interpret the highly asymmetric curved plumes evident in the maps as tracing the distribution of this material. Previous high-resolution efforts have been restricted to partially-resolved one-dimensional visibility curves interpreted in the context of spherically symmetric outflow models [7, 8]. As a comparison with these earlier results, we have fitted a uniform-disk model to our visibilities, azimuthally averaged and cropped to the resolutions then obtained, finding perfect agreement with the 130 mas diameter disk reported in 1981 [7]. This similarity over a timescale of decades is in accord with the inclusion of WR 104 in the small handful of 'persistent' dust producing WR's [9]. Additional interferometric observations at $3.08 \mu \mathrm{m}(\Delta \lambda=0.1 \mu \mathrm{m})$ show no evidence of the marked enlargement towards longer wavelengths reported by Dyck et. al. [8]. However, as 


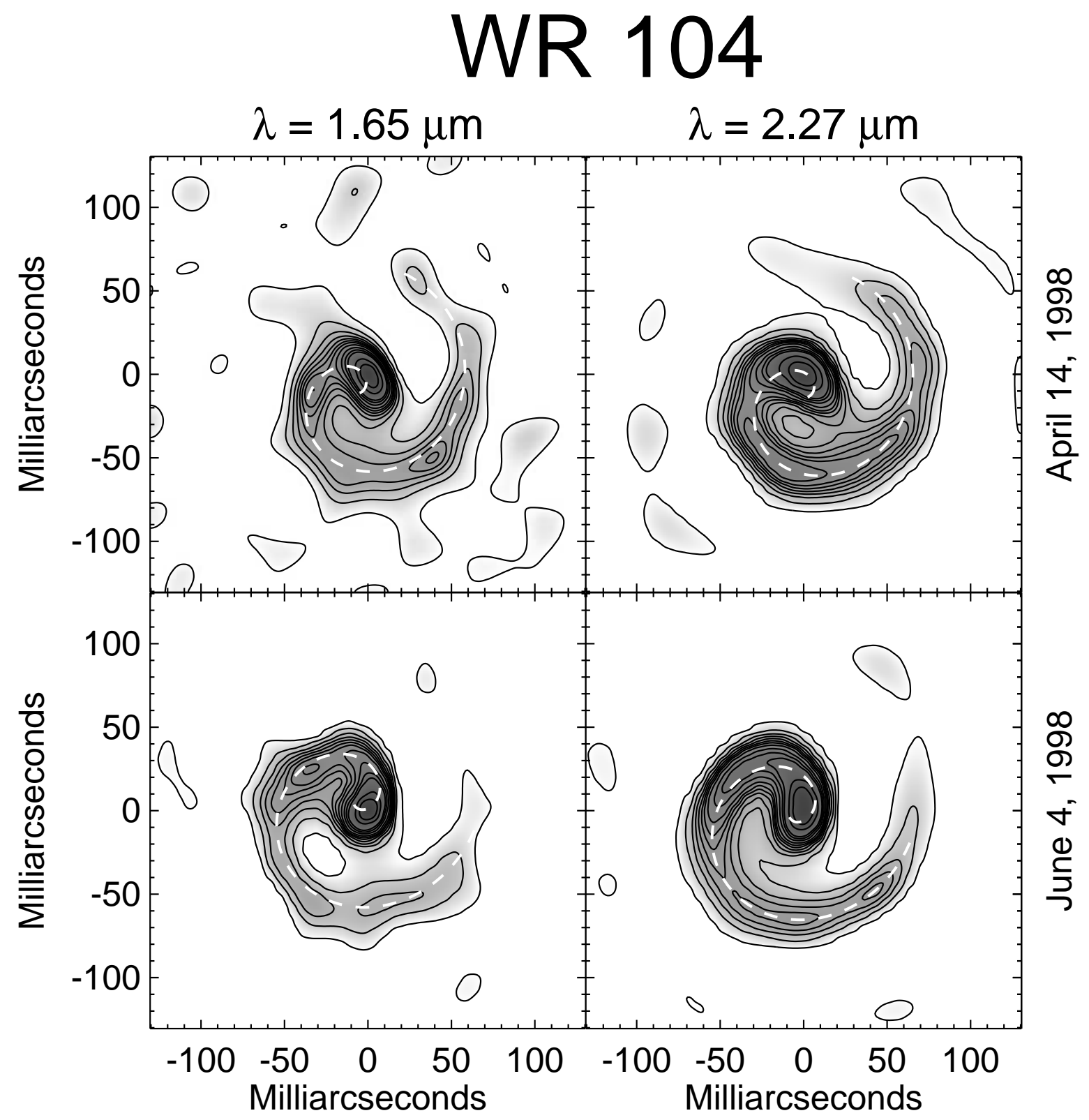

Figure 1: Maps of WR 104. Maximum-Entropy image reconstructions of WR 104 at 1.65 (left) and $2.27 \mu \mathrm{m}$ (right) taken over two separate epochs: April (upper) and June (lower) 1998 (JD 2450918 \& JD 2450969 respectively). Contour levels are 0.5, 2, 4, 6, 8, 10, 12, $15,20,25,30,50 \& 70 \%$ of the peak. Overplotted on each map is the best-fit Archimedian spiral model (dashed line). Observations utilized an annulus shaped pupil mask to form the interference pattern, which was recorded on a fast-readout $(130 \mathrm{~ms})$ infrared array and subsequently processed to extract Fourier amplitudes and closure phases. Bispectral information constituting about 700 baselines and 7000 closing triangles enabled high-quality images to be produced from an algorithm based on the maximum entropy method [5, 6]. Although these images are at the heart of our discussion, it is important to note that clear and systematic signals betraying the presence of the final image morphology are directly visible in the calibrated Fourier data themseles. 


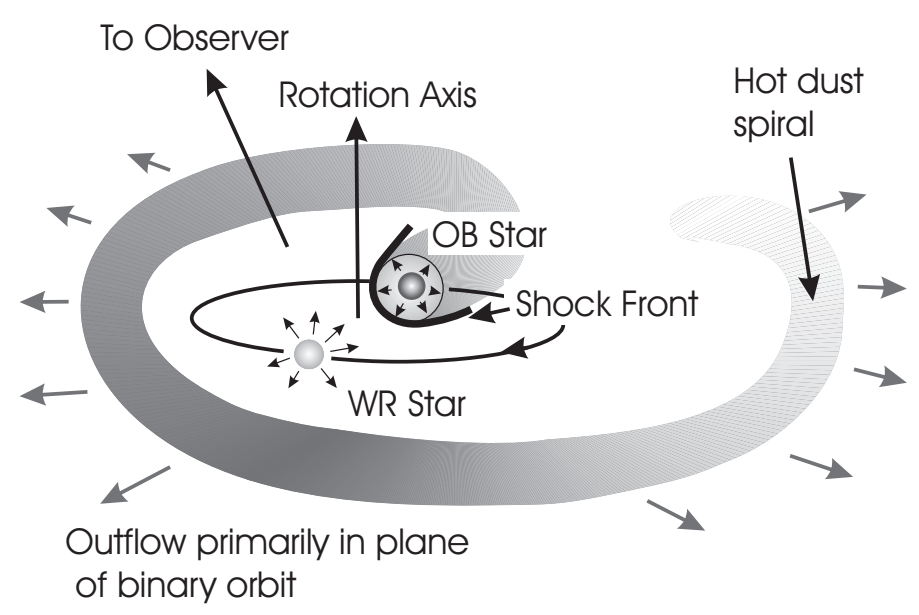

Figure 2: Schematic diagram of the WR 104 binary system. The illustration shows the WR star, the OB companion, wind-wind collision front, and the resultant dust outflow plume (not to scale). The spiral shape is a consequence of material being swept radially outwards by the WR wind from a rotating dust nucleation zone associated with the shock front where the stellar winds collide.

is apparent from Figure 1, the images do not show even remote similarity to a uniform disk, and we hereafter abandon further consideration of circularly symmetric models.

The maps of Figure 1 consist of two components; a bright central core which appears elongated, and a curved tail which seems to emerge from one end of the elongation. This spiral structure dominates the morphology at both colors, and maps taken in April and June 1998 show a high degree of similarity with the striking exception of a clear rotation of the image. The hypothesis of dust formation mediated by the orbital motion of a companion star and subsequently swept outwards by the stellar wind unifies the spiral structure and the $-83^{\circ}$ rotation apparent between our two epochs into a simple, elegant geometry. A schematic of our model is shown in Figure 2, showing the WR+OB binary, the dust formation zone associated with the collision front between the stellar winds [2], and the resultant curved outflow plume as this dust 'nursery' is carried with the orbital motion. Although the idea of a binary nature for WR 104 is not new, it is only very recently that the presence of an OB companion was confirmed from detection of hydrogen Balmer absorption features and optical emission-line dilution [10, 11]. 
We have overplotted, also in Figure 1, the results of fitting a simple geometrical model consisting of an Archimedian spiral where the free parameters are the winding rate and the viewing angle to the observer. These modelling results were obtained by finding a global best fit to all four maps simultaneously, but allowing for a rotation of the spiral structure about the model-derived axis between the two epochs. Implicit in the assumption of an Archimedian spiral model is the hypothesis that the material in the spiral is moving out at a uniform velocity, and that new material feeding into the flow insertion point does so at a uniform angular velocity. Although such a model contains the fewest free parameters and yet gives excellent fits to our data, it is important to note that a more complex model may be required if the plume is in a zone where it is being accelerated, or if the orbit of the companion presumed to be mediating the flow is eccentric (e.g. [9]).

The physical geometry of the system, as derived directly from our model, is a spiral plume rotating with a period of $220 \pm 30$ days viewed at an angle of $20 \pm 5^{\circ}$ from the pole and with an outflow velocity of $111 \pm 17$ mas yr$^{-1}$ in the plane of the orbit. If we identify this rotation as the orbital period of a binary stellar system, then assuming a combined mass in the range of $20-50 M_{\odot}$ [12] results in a separation of $1.9-2.6 \mathrm{AU}$. As this corresponds to a separation of only $\sim 1$ mas on the sky, our images lack the resolution to show such detail directly, and furthermore the infrared flux is so dominated by thermal emission from the warm dust [13] that it is unlikely that we have detected the central stars in our maps at all. It is interesting to compare our binary parameters with those of the famous 'episodic' dust producer WR 140 which is known to undergo dramatic bouts of dust creation coincident with the passage of a companion star through periastron in a highly elliptical orbit [9]. At periastron, the separation between the stars is $\sim 2.5 \mathrm{AU}$, raising the possibility that the physical conditions favoring copious dust formation fall within a confined range of companion distances for $\mathrm{WC}+\mathrm{OB}$ binaries.

We may make use of the $1220 \mathrm{~km} \mathrm{~s}^{-1}$ wind outflow velocity 14 combined with our proper motion to derive an independent estimate of $2.3 \pm 0.7 \mathrm{kpc}$ as the distance to WR 104, where the dominant error arises from a $\sim 25 \%$ uncertainty in outflow velocities found by comparing the results of various line-profile studies [15] (velocities as high as $1600 \mathrm{~km} \mathrm{~s}^{-1}$ have been reported for this star [16]). Our distance is somewhat further than earlier estimates of $1.6 \mathrm{kpc}$ derived from a possible association with Sgr OB1 [17], however the discrepancy is within the estimated errors. Alternatively if the closer distance is preferred, then our measurements imply an outflow velocity of $845 \mathrm{~km} \mathrm{~s}^{-1}$ for the dust component. Our geometrical solution solves for the projected viewing angle of the observer, and thus we avoid the usual $\sin (i)$ uncertainty. We note that although isolated dust grains should be momentum-coupled to the flow [1], the outflow velocities in the wake of the passage of the OB stellar companion could be significantly perturbed and thus the behavior of the plume may not act as a good tracer of the bulk motion of the stellar wind. With additional observations covering an entire orbit, we will be able to greatly refine our estimates of the physical geometry of this system.

It is apparent from Figure 1 that the outflowing material presents a relatively smooth, spatially confined stream without strong clumping out to a radius of some $\sim 65$ mas $(\sim$ $150 \mathrm{AU})$ from the star, by which time the outflow has rotated through about $360^{\circ}$. We 
believe that the finding of a single complete turn in the spiral arm is not coincidental, as we detect only dust heated by radiation from the central stars, and therefore lying along a direct line of sight. Material in the second and further coils of the outflow will, of course, be eclipsed by newer material closer in, and will therefore cool rapidly resulting in the relatively sharp cutoff we see. Although there is some evidence for brightness variations along the arm at a level of a few percent of the peak, especially apparent in the maps taken at $1.65 \mu \mathrm{m}$, the overall behavior points to a continuous and smooth dust creation process, in accord with the classification of WR 104 as a 'persistent' dust producer with a constant IR flux [18]. Again it is interesting to compare this behavior with that of WR 140 whose elliptical orbit results in episodic dust production. The contrasting characteristics of WR 104 argue against a high degree of orbital eccentricity, giving some justification to our choice of the Archimedian spiral model in this case.

For WR 104, our observations confine the IR excess emission from the dust to lie in a narrow, spatially confined outflow which rotates synchronously with a period of 220 days - a plausible period for a wind-interacting binary system. No spherically symmetric or diffuse component to the dust nebula was detected to within a few percent of the peak flux. We are therefore able to reject dust-formation models resulting in spherical or disk shaped outflows such as the clumpy spherical outflows of [19] or equatorial density enhancements [20] in favor of the binary wind-wind model.

The viewing angle to the observer of $20 \pm 5^{\circ}$ is well constrained by these measurements. This finding of an almost face-on system contradicts previous attribution of high circumstellar extinction [21] and spectral variability [11] to an edge-on viewing angle. Some of these observations may be explained as WR 104 is thought to lie behind a heavily obscuring cloud [17] with further extinction possibly arising from material created in past mass-loss events of the progenitor star.

As the dust comprises only a very small fraction of the total mass loss, it therefore acts as a visible tracer in the outflow enabling the fascinating possibility of dynamical studies of the wind itself. Detailed numerical modelling is needed to determine if the high degree of initial collimation and subsequent confinement of the dust plume can be explained with simple models of the wind-wind interaction [2], or whether more detailed three-dimensional calculations such as those of Walder [22], are required. Spectral studies of the plume, beyond the scope of this letter, should reveal the thermal and chemical evolution of the dust as it is swept outwards into the interstellar medium, and also yield information on processes underlying the binary-mediated dust creation mechanism. With a handful of dusty WR systems open to study with this novel method for the detection of binary stars, wider questions of dust formation in this class of objects can now be addressed.

\section{References}

[1] Williams, P.M., van der Hucht, K.A., and Thé, P.S., Infrared photometry of late-type Wolf-Rayet stars. Astron. Astrophys. 182 91-106 (1987) 
[2] Usov, V.V., Stellar wind collision and dust formation in long-period, heavily interacting Wolf-Rayet binaries. Mon. Not. R. Astron. Soc. 252 49-52 (1991)

[3] Haniff, C.A., \& Buscher, D.F., Diffraction-limited imaging with partially redundant masks - I. Infrared imaging of bright objects. J. Opt. Soc. Am. A, 9, 203-218 (1992)

[4] Tuthill, P.G., Monnier, J.D., Danchi, W.C., \& Haniff, C.A., Michelson Interferometry with Keck I. (in Proc SPIE 3350, Astronomical Interferometry, eds R.D. Reasenberg \& J.D. Breckinridge), P839-846 (1998)

[5] Gull, S.F., \& Skilling, J., Maximum entropy method in image processing. IEE Proceedings, 131, Pt F, No. 6. 646-650 (1984)

[6] Sivia, D.S., PhD Dissertation, Cambridge University (1987).

[7] Allen, D.A., Barton, J.R., and Wallace, P.T., The size of a Wolf-Rayet star's dust shell measured by speckle interferometry. Mon. Not. R. Astron. Soc. 196 797-800 (1981)

[8] Dyck, H.M., Simon, T., and Wolstencroft, R.D., The infrared dust shell around the WC9 star Ve 2-45. Astrophys J. 277 675-677 (1984)

[9] Williams, P.M., Formation of dust in hostile environments - what we learn from observing Wolf-Rayet stars. Astrophys \& Sp. Sci. 251 321-331 (1997)

[10] Williams, P.M. and van der Hucht K.A., A search for companions to dust-making WolfRayet stars in Wolf-Rayet stars in the framework of stellar evolution (eds Vreux J.-M et. al. ). 353-359 (Proc. 33rd Liège Int. Astrophys. Colloq, Univ Liège 1996)

[11] Crowther, P.A., Remarkable spectral variability in WR 104: dust condensation in a hostile environment? Mon. Not. R. Astron. Soc. 290 L59-L63 (1997)

[12] Moffat, A.F., Niemela, V.S., and Marraco, H.G., Wolf-Rayet stars in the magellanic clouds. VI. Spectroscopic orbits of WC binaries and implications for WR evolution. Astrophys J. 348 232-241 (1990)

[13] Zubko, V.G., On the physical model of dust around Wolf-Rayet stars. Mon. Not. R. Astron. Soc. 295 109-118 (1998)

[14] Howarth, I.D., and Schmutz, W., Near-infrared spectroscopy of Galactic Wolf-Rayet stars. Astron. Astrophys. 261 503-522 (1992)

[15] Rochowicz, K., and Nidezielski, A., Terminal velocities of Wolf-Rayet star winds from low resolution IUE spectra. Acta Astronomica 45 307-318 (1995)

[16] Torres, A.V., Conti, P.S., and Massey, P., Spectroscopic studies of Wolf-Rayet stars III. The WC subclass. Astrophys J. 300 379-395 (1986)

[17] Lundstrom, I., and Stenholm, B., Wolf-Rayet stars in open clusters and associations. Astron. Astrophys. Suppl. 58 163-192 (1984) 
[18] van der Hucht et. al. ISO SWS spectrophotometry of galactic Wolf-Rayet stars: preliminary results. Astron. Astrophys. 315 L193-L196 (1996)

[19] Veen, P.M. et. al. WR 121 obscured by a dust cloud: the key to understanding occasional "eclipses" of "dusty" Wolf-Rayet WC stars? Astron. Astrophys. 329 199-212 (1998)

[20] Cassinelli, J.P., Ignace, R., and Bjorkman, J.E., Winds from rotating Wolf-Rayet stars: the wind-compressed zone model. in Wolf-Rayet stars: binaries, colliding winds, evolution (eds van der Hucht, K.A., and Williams, P.R.). 191-202 (IAU Symp. No. 163, Kluwer, Dordrecht 1995)

[21] Cohen, M., Barlow, M.J., and Kuhi L.V., Wolf-Rayet stars. VI - The nature of the optical and infrared continua. Astron. Astrophys. 40 291-302 (1975)

[22] Walder, R., Simulations of colliding winds in three dimensions. in Wolf-Rayet stars: binaries, colliding winds, evolution (eds van der Hucht, K.A., and Williams, P.R.). 420-424 (IAU Symp. No. 163, Kluwer, Dordrecht 1995)

Acknowledgements Data herein were obtained at the W.M. Keck Observatory, made possible by the generous support of the W.M. Keck Foundatation, and operated as a scientific partnership among the California Institute of Technology, the University of California and NASA. This work was supported through grants from the National Science Foundation. The authors would like to thank Devinder Sivia for the maximum-entropy mapping program "VLBMEM" and David Hale for sparking our interest in Wolf-Rayet stars.

All correspondence should be addressed to Peter Tuthill (e-mail:gekko@ssl.berkeley.edu) 